\title{
SUB-SOLUTIONS AND MEAN-VALUE OPERATORS FOR ULTRAPARABOLIC EQUATIONS ON LIE GROUPS
}

\author{
CHIARA CINTI
}

\begin{abstract}
The aim of this paper is to provide a theory of sub-solutions for a class of hypoelliptic ultraparabolic operators $\mathscr{L}$, by using mean-value operators on the level sets of the fundamental solution of $\mathscr{L}$.
\end{abstract}

\section{Introduction}

In this paper we investigate several questions in Potential Theory related to a class of hypoelliptic ultraparabolic operators $\mathscr{L}$ with underlying homogeneous Lie group structures. Our class is contained in the one of the Hörmander operators and was singled out by Kogoj and Lanconelli in [9]. It contains, e.g., the heat operators on Carnot groups and the Kolmogorov type operators studied in [12]. We are mainly interested in a characterization of $\mathscr{L}$-subharmonic functions in terms of suitable mean-value operators and in representation formulas. We also characterize the bounded-above $\mathscr{L}$-subharmonic functions in $\mathrm{R}^{N+1}$ and their related $\mathscr{L}$-Riesz measures. These results extend to our class of operators several theorems proved by Watson in [14], [15], [16] for temperatures and subtemperatures. They also extend some results first proved in [7], [8] for classical parabolic operators with smooth coefficients.

We consider operators of the following type

$$
\mathscr{L}=\sum_{j=1}^{m} X_{j}^{2}+X_{0}-\partial_{t} \quad \text { in } \quad \mathrm{R}^{N+1},
$$

where the $X_{j}$ 's are smooth vector fields on $\mathbf{R}^{N}$, i.e. denoting $z=(x, t)$ the point in $\mathbf{R}^{N+1}$

$$
X_{j}(x)=\sum_{k=1}^{N} a_{j}^{k}(x) \partial_{x_{k}}, \quad j=0, \ldots, m,
$$

Received August 29, 2005. 
where any $a_{j}^{k}$ is a $C^{\infty}$ function. For our purposes, in the sequel we also consider the $X_{j}$ 's as vector fields in $\mathrm{R}^{N+1}$. We denote by $Y$ the vector field in $\mathrm{R}^{N+1}$

$$
Y:=X_{0}-\partial_{t},
$$

and by $\mathscr{L}_{0}$ the operator in $\mathrm{R}^{N}$

$$
\mathscr{L}_{0}:=\sum_{j=1}^{m} X_{j}^{2}+X_{0}
$$

We next state our main assumptions:

(H.1) there exists a homogeneous Lie group $\mathrm{L}=\left(\mathrm{R}^{N+1}, \circ, d_{\lambda}\right)$ such that

(i) $X_{1}, \ldots, X_{m}, Y$ are left invariant on $\mathrm{L}$;

(ii) $X_{1}, \ldots, X_{m}$ are $d_{\lambda}$-homogeneous of degree one and $Y$ is $d_{\lambda}$ homogeneous of degree two;

(H.2) for every $(x, t),(\xi, \tau) \in \mathrm{R}^{N+1}$ with $t>\tau$, there exists an $\mathscr{L}$ admissible path $\eta:[0, T] \longrightarrow \mathrm{R}^{N+1}$ such that $\eta(0)=(x, t)$, $\eta(T)=(\xi, \tau)$. The curve $\eta$ is called $\mathscr{L}$-admissible if it is absolutely continuous and satisfies

$$
\eta^{\prime}(s)=\sum_{j=1}^{m} \lambda_{j}(s) X_{j}(\eta(s))+\lambda_{0}(s) Y(\eta(s)), \quad \text { a.e. in } \quad[0, T],
$$

for suitable piecewise constant real functions $\lambda_{0}, \lambda_{1}, \ldots \lambda_{m}, \lambda_{0} \geq 0$.

The operators of the form (1.1) with the assumptions (H.1) and (H.2) have been introduced by Kogoj and Lanconelli in [9].

The aim of this paper is to provide a theory of $\mathscr{L}$-subharmonic functions by using mean-value operators on the level sets of $\Gamma$, the fundamental solution of $\mathscr{L}$. More precisely, the contents of the paper are the following ones. After collecting in Section 2 some basic results of Potential Theory for $\mathscr{L}$, in Section 3 we recall some mean-value representation formulas and we present some properties of $\mathscr{L}$-harmonic functions with respect to mean-value integral operators $\mathscr{M}_{r}$ and $M_{r}$. In Section 4, we show some results on upper semi-continuous functions satisfying solid sub-mean property. In Section 5, we prove some characterizations of $\mathscr{L}$-subharmonic functions in terms of averaging operators $\mathscr{M}_{r}$ and $M_{r}$. Finally, in Section 6 we give a characterization of the bounded-above $\mathscr{L}$-subharmonic functions in $\mathrm{R}^{N+1}$ and their related $\mathscr{L}$-Riesz measures. 


\section{Basic potential theory for $\mathscr{L}$}

We first recall some consequences of hypotheses (H.1) and (H.2). One of these is the Hörmander condition:

$$
\operatorname{rank} \operatorname{Lie}\left\{X_{1}, \ldots, X_{m}, Y\right\}(z)=N+1, \quad \forall z \in \mathrm{R}^{N+1} ;
$$

hence $\mathscr{L}$ and $\mathscr{L}_{0}$ are hypoelliptic operators in $\mathrm{R}^{N+1}$ and in $\mathrm{R}^{N}$ respectively (see [9, Proposition 10.1]). From (H.1) and (H.2) it follows also that the composition law o is euclidean in the "time" variable, i.e.

$$
(x, t) \circ(\xi, \tau)=(S(x, t, \xi, \tau), t+\tau)
$$

for a suitable smooth function $S$, and the dilation $d_{\lambda}$ takes the following form

$$
d_{\lambda}(x, t)=\left(D_{\lambda}(x), \lambda^{2} t\right)=\left(\lambda^{\sigma_{1}} x_{1}, \ldots, \lambda^{\sigma_{N}} x_{N}, \lambda^{2} t\right) .
$$

The natural number

$$
Q=\sum_{k=1}^{N} \sigma_{k}+2
$$

is the homogeneous dimension of $\mathrm{L}$. We shall assume that $Q \geq 5$ so that $Q-2$, the homogeneous dimension of $\mathrm{R}^{N}$ with respect to $D_{\lambda}$, will be $\geq 3$. We shall denote by $|\cdot|$ a fixed $d_{\lambda}$-homogeneous norm on $\mathrm{L}$, that is a function $|\cdot|: \mathrm{R}^{N+1} \longrightarrow\left[0, \infty\left[\right.\right.$ with the following properties: $|\cdot| \in C^{\infty}\left(\mathrm{R}^{N+1} \mid\right.$ $\{(0,0)\}) \cap C\left(\mathrm{R}^{N+1}\right),\left|d_{\lambda}(z)\right|=\lambda|z|,\left|z^{-1}\right|=|z|,|z|=0$ iff $z=0$.

In [9] it is proved that $\mathscr{L}$ has a global fundamental solution $\Gamma \in C^{\infty}\left(\mathrm{R}^{N+1} \backslash\right.$ $\{(0,0)\})$ such that $\mathscr{L} \Gamma=-\delta$. Moreover $\Gamma(x, t)>0$ iff $t>0$. If we put

$$
\Gamma(z, \zeta):=\Gamma\left(\zeta^{-1} \circ z\right),
$$

since $\mathscr{L}$ is left translation invariant, we have $\mathscr{L} \Gamma(\cdot, \zeta)=-\delta_{\zeta}$ for every $\zeta \in \mathrm{R}^{N+1}$.

Integrating $\Gamma$ with respect to the $t$ variable one obtains a fundamental solution $\gamma$ with pole at $x=0$ for the operator $\mathscr{L}_{0}$ (see [9, Section 3]):

$$
\gamma(x):=\int_{0}^{\infty} \Gamma(x, t) \mathrm{d} t
$$

$\gamma$ is smooth and strictly positive out of the origin.

Throughout the paper, $\Omega$ will always denote an open subset of $\mathrm{R}^{N+1}$, even if we do not mention this. We call $\mathscr{L}$-harmonic in $\Omega$ every smooth function $u: \Omega \longrightarrow \mathrm{R}$ such that $\mathscr{L} u=0$. We shall denote by $\mathscr{H}^{\mathscr{L}}(\Omega)$ the linear space of $\mathscr{L}$-harmonic functions in $\Omega$. 
We say that a bounded open set $V \subset \mathrm{R}^{N+1}$ is $\mathscr{L}$-regular if for any $\varphi \in$ $C(\partial V)$ there exists a unique function $H_{\varphi}^{V} \in \mathscr{H}^{\mathscr{L}}(V)$ such that

$$
\lim _{z \rightarrow z_{0}} H_{\varphi}^{V}(z)=\varphi\left(z_{0}\right), \quad \text { for every } \quad z_{0} \in \partial V,
$$

and $H_{\varphi}^{V} \geq 0$ whenever $\varphi \geq 0$, as the classical Picone's maximum principle holds for $\mathscr{L}$ (see [9, Proposition 2.1]). Then, if $V$ is $\mathscr{L}$-regular, for every fixed $z \in V$ the map

$$
C(\partial V) \ni \varphi \mapsto H_{\varphi}^{V}(z) \in \mathrm{R}
$$

defines a linear positive functional on $C(\partial V)$. As a consequence, there exists a Radon measure $\mu_{z}^{V}$ supported in $\partial V$, such that

$$
H_{\varphi}^{V}(z)=\int_{\partial V} \varphi(\zeta) \mathrm{d} \mu_{z}^{V}(\zeta), \quad \text { for every } \varphi \in C(\partial V)
$$

We call $\mu_{z}^{V}$ the $\mathscr{L}$-harmonic measure related to $V$ and $z$.

We say that $u: \Omega \longrightarrow\left[-\infty, \infty\left[\right.\right.$ is $\mathscr{L}$-subharmonic in $\Omega\left(u \in \underline{\mathscr{S}}^{\mathscr{L}}(\Omega)\right)$ if $u$ is upper semi-continuous (u.s.c.), $u>-\infty$ in a dense subset of $\Omega$, and for every open $\mathscr{L}$-regular set $V \subset \bar{V} \subset \Omega$ and for every $z \in V$,

$$
u(z) \leq \int_{\partial V} u(\zeta) \mathrm{d} \mu_{z}^{V}(\zeta)
$$

It is easy to prove that a function $u: \Omega \longrightarrow[-\infty, \infty[$ u.s.c. and finite in a dense subset of $\Omega$ is $\mathscr{L}$-subharmonic in $\Omega$ if

$$
u \leq H_{\varphi}^{V} \quad \text { in } \quad V,
$$

for every $V$ open $\mathscr{L}$-regular set, $\bar{V} \subset \Omega$, and for every $\varphi \in C(\partial V)$ such that $\varphi \geq\left. u\right|_{\partial V}$. Proceeding as in [13, Theorem 1], we can obtain the following further characterization of $\mathscr{L}$-subharmonic functions.

Proposition 2.1. Let $u: \Omega \longrightarrow[-\infty, \infty[$ be an u.s.c. function. Then, if $u \in \underline{\mathscr{S}}^{\mathscr{L}}(\Omega)$, we have $u \in L_{\text {loc }}^{1}(\Omega)$ and $\mathscr{L} u \geq 0$ in the distribution sense.

Remark 2.2. By Proposition 2.1, if $u \in \underline{\mathscr{S}}^{\mathscr{L}}(\Omega)$ then there exists a Radon measure $\mu$ in $\Omega$ such that $\mathscr{L} u=\mu$. We shall call $\mu$ the $\mathscr{L}$-Riesz measure related to $u$.

We obviously have $\underline{\mathscr{S}}^{\mathscr{L}}(\Omega) \cap\left(-\underline{\mathscr{S}}^{\mathscr{L}}(\Omega)\right)=\mathscr{H}^{\mathscr{L}}(\Omega)$. 
In the sense of the abstract Potential Theory (see, e.g., [6]), the map $\mathrm{R}^{N+1} \supseteq$ $\Omega \mapsto \mathscr{H}^{\mathscr{L}}(\Omega)$ is a harmonic sheaf and $\left(\mathrm{R}^{N+1}, \mathscr{H}^{\mathscr{L}}\right)$ is a $\mathfrak{B}$-harmonic space. The second statement is a consequence of the following properties:

- the $\mathscr{L}$-regular sets form a basis of the Euclidean topology (see [5, Corollary 5.2]);

- $\mathscr{H}^{\mathscr{L}}$ satisfies the Doob convergence property, i.e. the pointwise limit of any increasing sequence of $\mathscr{L}$-harmonic functions on any open set is $\mathscr{L}$-harmonic whenever it is finite on a dense set (see [9, Proposition 7.4]);

- for every fixed $\zeta=(\xi, \tau) \in \mathrm{R}^{N+1}$, the functions $z \mapsto-\Gamma\left(\zeta^{-1} \circ z\right)$ and $(x, t) \mapsto-\gamma\left(\xi^{-1} \circ x\right)$ are $\mathscr{L}$-subharmonic in $\mathrm{R}^{N+1}$ and it easy to show that the families $\left\{z \mapsto-\Gamma\left(\zeta^{-1} \circ z\right) \mid \zeta \in \mathrm{R}^{N+1}\right\},\{(x, t) \mapsto$ $\left.-\gamma\left(\xi^{-1} \circ x\right) \mid \xi \in \mathbf{R}^{N}\right\}$ separate the points of $\mathbf{R}^{N+1}$.

\section{Mean-value formulas and $\mathscr{L}$-harmonic functions}

Given $z \in \mathrm{R}^{N+1}$ and $r>0$, we define the $\mathscr{L}$-ball of center $z$ and radius $r$ as follows:

$$
\Omega_{r}(z):=\left\{\zeta \in \mathrm{R}^{N+1} \mid \Gamma\left(\zeta^{-1} \circ z\right)>\frac{1}{r^{Q-2}}\right\} .
$$

Obviously, $\Omega_{r}(z)=z \circ \Omega_{r}(0)$. The properties of the $\mathscr{L}$-balls stated in the next proposition directly follow from the properties of the fundamental solution $\Gamma$ proved in [9].

Proposition 3.1. For every $z \in \mathrm{R}^{N+1}$, the $\mathscr{L}$-balls centered in $z$ have the following properties:

(i) for every $r>0, \Omega_{r}(z)$ is a bounded nonempty set;

(ii) $\Omega_{r}(z)$ shrinks to $\{z\}$ as $r$ goes to 0 , that is $\bigcap_{r>0} \bar{\Omega}_{r}(z)=\{z\}$;

(iii) if we denote by $\left|\Omega_{r}(z)\right|$ the Lebesgue measure of $\Omega_{r}(z)$, then

$$
\lim _{r \rightarrow 0^{+}} \frac{\left|\Omega_{r}(z)\right|}{r^{Q-2}}=0
$$

(iv) for almost every $r>0, \partial \Omega_{r}(z)$ is a $N$-dimensional $C^{\infty}$ manifold;

(v) if $z=(x, t)$, then $\left.\bigcup_{r>0} \Omega_{r}(z)=\mathrm{R}^{N} \times\right]-\infty, t[$.

If $\Omega \subseteq \mathrm{R}^{N+1}$ is an open set containing 0 , and $v \in C^{2}(\Omega)$, we have

$$
v(0)=\mathscr{M}_{r}(v)(0)-\mathcal{N}_{r}(\mathscr{L} v)(0), \quad \text { for every } \quad \bar{\Omega}_{r}(0) \subseteq \Omega ;
$$


where

$$
\begin{aligned}
\mathscr{M}_{r}(v)(0) & :=\int_{\partial \Omega_{r}(0)} \mathscr{K}(\zeta) v(\zeta) \mathrm{d} \sigma(\zeta), \quad \text { with } \quad \mathscr{K}(\zeta):=\frac{\left|\nabla_{\mathscr{L}} \Gamma(0, \zeta)\right|^{2}}{\left|\nabla_{\zeta} \Gamma(0, \zeta)\right|} \\
\mathscr{N}_{r}(\mathscr{L} v)(0) & :=\int_{\Omega_{r}(0)}\left(\Gamma(0, \zeta)-\frac{1}{r^{Q-2}}\right) \mathscr{L} v(\zeta) \mathrm{d} \zeta .
\end{aligned}
$$

Hereafter we denote by $\nabla_{\mathscr{L}}$ the vector valued differential operator

$$
\nabla_{\mathscr{L}}=\left(X_{1}, \ldots, X_{m}\right),
$$

and $\nabla_{x}=\left(\partial_{x_{1}}, \ldots, \partial_{x_{N}}\right)$.

Formula (3.1) is a particular version of the Green representation theorem for $\mathscr{L}$. In order to get it, we proceed as in [11, Theorem 1.5], using the properties of fundamental solution $\Gamma$ showed in [9], in particular the inequality (5.1) and the identity (6.1), and writing $\mathscr{L}$ in the following divergence form

$$
\mathscr{L}=\operatorname{div}\left(A \nabla_{x}\right)+Y,
$$

where $A$ is a suitable $N \times N$ matrix, and $Y$ is divergence free.

Let $z \in \mathrm{R}^{N+1}$. We apply (3.1) at the function $v_{z}(\zeta)=u(z \circ \zeta)$, and using the invariance of $\mathscr{L}$ w.r.t. the left translations on $\mathrm{L}$ we get

$$
\begin{aligned}
u(z) & =\int_{\partial \Omega_{r}(0)} \mathscr{K}(\zeta) u(z \circ \zeta) \mathrm{d} \sigma(\zeta)-\int_{\Omega_{r}(z)}\left(\Gamma\left(\zeta^{-1} \circ z\right)-\frac{1}{r^{Q-2}}\right) \mathscr{L} u(\zeta) \mathrm{d} \zeta \\
& =: \mathscr{M}_{r}(u)(z)-\mathscr{N}_{r}(\mathscr{L} u)(z), \quad \text { for every } \quad \bar{\Omega}_{r}(z) \subseteq z \circ \Omega .
\end{aligned}
$$

Setting $r=l$ in (3.2), multiplying both sides by $l^{Q-3}$ and integrating between 0 and $r$ give

$$
u(z) \frac{r^{Q-2}}{Q-2}=\int_{0}^{r} l^{Q-3} \mathscr{M}_{l}(u)(z) \mathrm{d} l-\int_{0}^{r} l^{Q-3} \mathcal{N}_{l}(\mathscr{L} u)(z) \mathrm{d} l,
$$

then, by means of Federer's co-area formula, we obtain

$$
\begin{aligned}
u(z) & =\frac{1}{r^{Q-2}} \int_{\Omega_{r}(z)} K\left(\zeta^{-1} \circ z\right) u(\zeta) \mathrm{d} \zeta-\frac{Q-2}{r^{Q-2}} \int_{0}^{r} l^{Q-3} \mathcal{N}_{l}(\mathscr{L} u)(z) \mathrm{d} l \\
& =: M_{r}(u)(z)-N_{r}(\mathscr{L} u)(z), \quad \text { for every } \quad \bar{\Omega}_{r}(z) \subseteq z \circ \Omega,
\end{aligned}
$$

where

$$
K\left(\zeta^{-1} \circ z\right)=K(z, \zeta):=\frac{\left|\nabla_{\mathscr{L}} \Gamma(z, \zeta)\right|^{2}}{\Gamma^{2}(z, \zeta)} .
$$


We explicitly note that the kernel $K$ is invariant w.r.t. the left translation on L, unlike $\mathscr{K}$. Let $z=(x, t)$ be fixed. We have $K(z, \cdot) \geq 0$ in $\mathrm{R}^{N+1}, K(z, \cdot) \in$ $C^{\infty}\left(\left\{(\xi, \tau) \in \mathrm{R}^{N+1} \mid \tau<t\right\}\right)$. By [9, Lemma 7.3], the set

$$
\Sigma:=\left\{\zeta=(\xi, \tau) \in \mathrm{R}^{N+1} \mid \tau<t, K(z, \zeta)=0\right\}
$$

does not contain interior points.

Now, let $\Omega \subseteq \mathrm{R}^{N+1}$ be an arbitrary open set. By comparing (3.3) with (3.4), we deduce that, if $u \in C^{2}(\Omega)$,

$$
M_{r}(u)(z)=\frac{Q-2}{r^{Q-2}} \int_{0}^{r} l^{Q-3} M_{l}(u)(z) \mathrm{d} l, \quad \text { for every } \quad \bar{\Omega}_{r}(z) \subseteq \Omega .
$$

We stress that, by a standard argument of approximation, (3.5) also holds if $u$ is u.s.c.

Moreover, from (3.2) and (3.4) it follows that any $\mathscr{L}$-harmonic function in $\Omega$ satisfies the mean value formulas

$$
u(z)=M_{r}(u)(z) \quad \text { and } \quad u(z)=M_{r}(u)(z),
$$

for every $z \in \Omega$ and $r>0$ such that $\bar{\Omega}_{r}(z) \subseteq \Omega$. If $u \in C(\Omega)$, also the converse implication of this result is true. Indeed we have the following generalization to the classical Koebe theorem.

THeOREM 3.2. Let $u \in C(\Omega)$ be such that

$$
u(z)=\mathscr{M}_{r}(u)(z), \quad \text { for every } \bar{\Omega}_{r}(z) \subseteq \Omega .
$$

Then $u \in C^{\infty}(\Omega)$ and $\mathscr{L} u=0$. An analogous result holds if

$$
u(z)=M_{r}(u)(z), \quad \text { for every } \bar{\Omega}_{r}(z) \subseteq \Omega .
$$

In order to prove this theorem, we need a lemma. Let $J \in C_{0}^{\infty}\left(\mathrm{R}^{N+1}\right)$, $J \geq 0$ be such that supp $J \subseteq B(0,1)$ and $\int_{\mathrm{R}^{N+1}} J=1$. Let $\Omega \subseteq \mathrm{R}^{N+1}$ be an open set, and let $u \in L_{\text {loc }}^{1}(\Omega)$. For $\varepsilon>0$, we define the $\varepsilon$ - $\mathscr{L}$-mollified of $u$ in $\Omega$ as follows

$$
\begin{aligned}
u_{\varepsilon}: D_{\varepsilon}^{\Omega} & \rightarrow \mathrm{R} \\
z & \mapsto \int_{\Omega} u(\zeta) J\left(d_{\varepsilon^{-1}}\left(z \circ \zeta^{-1}\right)\right) \varepsilon^{-Q} \mathrm{~d} \zeta,
\end{aligned}
$$

where $D_{\varepsilon}^{\Omega}=\left\{\zeta \in \mathrm{R}^{N+1} \mid \bar{B}\left(\zeta^{-1}, \varepsilon\right) \subset \Omega^{-1}\right\}$. It is a standard matter to show that $u_{\varepsilon} \in C^{\infty}\left(D_{\varepsilon}^{\Omega}\right)$, and $u_{\varepsilon} \longrightarrow u$ in $L_{\mathrm{loc}}^{1}(\Omega)$ as $\varepsilon \rightarrow 0$. We next prove the so called solid sub-mean property of $u_{\varepsilon}$ (see Section 4 ). 
Lemma 3.3. Let $u: \Omega \longrightarrow\left[-\infty, \infty\left[\right.\right.$ be an u.s.c. function, $u \in L_{\mathrm{loc}}^{1}(\Omega)$. If $u(z) \leq M_{r}(u)(z)$ for every $\bar{\Omega}_{r}(z) \subseteq \Omega$, then $u_{\varepsilon}(z) \leq M_{r}\left(u_{\varepsilon}\right)(z)$ for every $\bar{\Omega}_{r}(z) \subseteq D_{\varepsilon}^{\Omega}$.

Proof. For $\bar{\Omega}_{r}(z) \subseteq D_{\varepsilon}^{\Omega}$, we have

$$
\begin{aligned}
& M_{r}\left(u_{\varepsilon}\right)(z) \\
& \quad=\int_{\bar{B}(0, \varepsilon)} J\left(d_{\varepsilon^{-1}}(\eta)\right) \varepsilon^{-Q}\left(\frac{1}{r^{Q-2}} \int_{\Omega_{r}(z)} K\left(\zeta^{-1} \circ z\right) u\left(\eta^{-1} \circ \zeta\right) \mathrm{d} \zeta\right) \mathrm{d} \eta \\
& \quad=\int_{\bar{B}(0, \varepsilon)} J\left(d_{\varepsilon^{-1}}(\eta)\right) \varepsilon^{-Q} M_{r}(u)\left(\eta^{-1} \circ z\right) \mathrm{d} \eta \\
& \quad\left(\bar{\Omega}_{r}\left(\eta^{-1} \circ z\right) \subseteq \bar{B}(0, \varepsilon) \circ D_{\varepsilon}^{\Omega} \subseteq \Omega\right) \\
& \geq \int_{\bar{B}(0, \varepsilon)} J\left(d_{\varepsilon^{-1}}(\eta)\right) \varepsilon^{-Q} u\left(\eta^{-1} \circ z\right) \mathrm{d} \eta=u_{\varepsilon}(z),
\end{aligned}
$$

and the assertion follows.

Proof of Theorem 3.2. It easily follows from (3.5) that (3.6) is equivalent to (3.7). Now, if $u \in C^{\infty}(\Omega)$ satisfies (3.6), then from (3.2) we obtain

$$
0=\mathcal{N}_{r}(\mathscr{L} u)(z)=\int_{\Omega_{r}(z)}\left(\Gamma\left(\zeta^{-1} \circ z\right)-\frac{1}{r^{Q-2}}\right) \mathscr{L} u(\zeta) \mathrm{d} \zeta
$$

and so $\mathscr{L} u=0$. Then, it suffices to prove that $u$ is smooth. If we show that $\mathscr{L} u=0$ in the distribution sense on $\Omega$, the assertion follows from the hypoellipticity of $\mathscr{L}$. From (3.7) and Lemma 3.3, we get $u_{\varepsilon}(z)=M_{r}\left(u_{\varepsilon}\right)(z)$ for every $\bar{\Omega}_{r}(z) \subseteq D_{\varepsilon}^{\Omega}$. So the sequence of $(1 / n)-\mathscr{L}$-mollified $\left\{u_{1 / n}\right\}_{n}$ is such that $\mathscr{L} u_{1 / n}=0$ in $D_{1 / n}^{\Omega}$ and $u_{1 / n} \longrightarrow u$ per $n \rightarrow \infty$ uniformly on compact subsets of $\Omega$. For every $\varphi \in C_{0}^{\infty}(\Omega)$, we have

$$
\int_{\Omega} u(\zeta) \mathscr{L}^{*} \varphi(\zeta) \mathrm{d} \zeta=\lim _{n \rightarrow \infty} \int_{D_{1 / n}^{\Omega} \cap \operatorname{supp} \varphi} \mathscr{L} u_{1 / n}(\zeta) \varphi(\zeta) \mathrm{d} \zeta=0
$$

and the smoothness of $u$ is proved.

We also show another property of the $\varepsilon$ - $\mathscr{L}$-mollified.

Proposition 3.4. Let $u \in \underline{\mathscr{S}}^{\mathscr{L}}(\Omega)$. Then $u_{\varepsilon}$ is $\mathscr{L}$-subharmonic in $D_{\varepsilon}^{\Omega}$.

Proof. Since $u_{\varepsilon} \in C^{\infty}\left(D_{\varepsilon}^{\Omega}\right)$, it is enough to prove that $\mathscr{L} u_{\varepsilon} \geq 0$ in the 
weak sense of distribution. Let $\varphi \in C_{0}^{\infty}\left(D_{\varepsilon}^{\Omega}\right), \varphi \geq 0$. We have

$$
\begin{aligned}
\left\langle\mathscr{L} u_{\varepsilon}, \varphi\right\rangle & =\int_{D_{\varepsilon}^{\Omega}} u_{\varepsilon}(z) \mathscr{L}^{*} \varphi(z) \mathrm{d} z \\
& =\int_{\bar{B}(0, \varepsilon)}\left(\int_{D_{\varepsilon}^{\Omega}} u\left(\eta^{-1} \circ z\right) \mathscr{L}^{*} \varphi(z) \mathrm{d} z\right) J\left(d_{\varepsilon^{-1}}(\eta)\right) \varepsilon^{-Q} \mathrm{~d} \eta \\
& =\int_{\bar{B}(0, \varepsilon)}\left(\int_{\Omega} u(\zeta) \mathscr{L}^{*}[\varphi(\eta \circ \zeta)] \mathrm{d} \zeta\right) J\left(d_{\varepsilon^{-1}}(\eta)\right) \varepsilon^{-Q} \mathrm{~d} \eta \geq 0,
\end{aligned}
$$

and the assertion is proved.

As a straightforward consequence, we have the following smoothing result.

Corollary 3.5. Let $u \in \underline{\mathscr{S}}^{\mathscr{L}}(\Omega)$. There exists a sequence of smooth $\mathscr{L}$-subharmonic functions which tends to $u$ in $L_{\text {loc }}^{1}(\Omega)$.

\section{Sub-mean functions}

We say that an u.s.c. function $u: \Omega \longrightarrow[-\infty, \infty[$ satisfies the surface (solid) sub-mean property if

$$
u(z) \leq \mathscr{M}_{r}(u)(z) \quad\left(u(z) \leq M_{r}(u)(z)\right), \quad \text { for every } \quad \bar{\Omega}_{r}(z) \subseteq \Omega .
$$

Next theorem shows that solid sub-mean functions satisfy a weak maximum principle.

THEOREM 4.1. Let $u: \Omega \longrightarrow[-\infty, \infty[$ be an u.s.c. function satisfying the solid sub-mean property. We have:

(i) if $\Omega$ is bounded and $\lim \sup _{\Omega \ni z \rightarrow \zeta} u(z) \leq 0$ for every $\zeta \in \partial \Omega$ then $u \leq 0$ in $\Omega$;

(ii) if $\Omega$ is unbounded and

$$
\limsup _{\Omega \ni z \rightarrow \zeta} u(z) \leq 0 \quad \text { for every } \zeta \in \partial \Omega, \quad \limsup _{z \in \Omega,|z| \rightarrow \infty} u(z) \leq 0,
$$

then $u \leq 0$ in $\Omega$.

Proof. (i) Let $z_{0} \in \bar{\Omega}$ be such that $\sup _{\Omega} u=\sup _{\Omega \cap V} u$ for every $V \in \mathscr{U}_{z_{0}}$, where $\mathscr{U}_{z_{0}}$ is the set of all the neighborhoods of $z_{0}$. If $z_{0} \in \partial \Omega$, by the hypothesis we have

$$
0 \geq \limsup _{\Omega \ni z \rightarrow z_{0}} u(z)=\inf _{V \in \mathscr{U}_{z_{0}}} \sup _{\Omega \cap\left(V \backslash\left\{z_{0}\right\}\right)} u=\inf _{V \in \mathscr{U}_{z_{0}}} \sup _{\Omega \cap V} u=\sup _{\Omega} u,
$$

whence $u \leq 0$ on $\Omega$. 
Let us suppose $z_{0} \in \Omega$. By the upper semicontinuity of $u, u\left(z_{0}\right)=$ $\inf _{V \in \mathscr{U}_{z 0}} \sup _{\Omega \cap V} u=\sup _{\Omega} u$, whence $u\left(z_{0}\right)=\max _{\Omega} u$. We may consider $u\left(z_{0}\right) \neq-\infty$, otherwise the claim is obvious. Since $\Omega$ is an open set, there exists $r>0$ such that $\bar{\Omega}_{r}\left(z_{0}\right) \subseteq \Omega$. By the solid sub-mean property of $u$,

$$
0 \leq \frac{1}{r^{Q-2}} \int_{\Omega_{r}\left(z_{0}\right)}\left(u(\zeta)-u\left(z_{0}\right)\right) K\left(\zeta^{-1} \circ z_{0}\right) \mathrm{d} \zeta .
$$

Thus, as $K\left(z_{0}, \cdot\right) \geq 0$ in $\mathrm{R}^{N+1}$ and $u(\zeta) \leq u\left(z_{0}\right)$,

$$
K\left(\zeta^{-1} \circ z_{0}\right)\left(u(\zeta)-u\left(z_{0}\right)\right)=0 \quad \text { a.e. in } \quad \Omega_{r}\left(z_{0}\right) .
$$

On the other hand, $K\left(z_{0}, \cdot\right)>0$ in a dense open subset of $\left\{(x, t) \in \mathrm{R}^{N+1}\right.$ $\left.t<t_{0}\right\}$ and $u$ is a u.s.c. function which attains in $z_{0}$ the maximum on $\Omega$. This yields

$$
u \equiv u\left(z_{0}\right) \text { on } \Omega_{r}\left(z_{0}\right), \quad \text { for every } \quad \bar{\Omega}_{r}\left(z_{0}\right) \subseteq \Omega .
$$

As $\Omega$ is bounded and by (4.2), it is easy to show that there exists $\left.r_{0} \in\right] 0, \infty[$ such that $u \equiv u\left(z_{0}\right)$ on $\Omega_{r_{0}}\left(z_{0}\right) \subseteq \Omega$, with $\bar{\Omega}_{r_{0}}\left(z_{0}\right) \nsubseteq \Omega$. Hence, for $\zeta \in$ $\bar{\Omega}_{r_{0}}\left(z_{0}\right) \cap \partial \Omega$, we have

$$
0 \geq \limsup _{\Omega \ni z \rightarrow \zeta} u(z) \geq \limsup _{\Omega_{r_{0}}\left(z_{0}\right) \ni z \rightarrow \zeta} u(z)=u\left(z_{0}\right)=\max _{\Omega} u,
$$

and this prove (i).

(ii) By the hypothesis, for every $\varepsilon>0$ there exists $R_{\varepsilon}>0$ such that

$$
\sup _{\Omega \backslash B(0, R)} u \leq \varepsilon, \quad \text { for every } \quad R \geq R_{\varepsilon} .
$$

We consider the bounded open set $\Omega_{R}:=\Omega \cap B(0, R)$ for $R \geq R_{\varepsilon}+1$, and let $\zeta \in \partial \Omega_{R}$. If $\zeta \in \partial \Omega \cap \bar{B}(0, R)$, it follows from the hypothesis that $\lim \sup _{\Omega_{R} \ni z \rightarrow \zeta} u(z) \leq \varepsilon$. Otherwise, if $\zeta \in \partial B(0, R) \cap \bar{\Omega}$, recalling (4.3) we obtain

$$
\limsup _{\Omega_{R} \ni z \rightarrow \zeta} u(z)=\inf _{V \in \mathscr{U}_{\zeta}} \sup _{V \cap \Omega_{R}} u \leq \sup _{\left(\Omega \backslash B\left(0, R_{\varepsilon}\right)\right) \cap \Omega_{R}} u \leq \varepsilon .
$$

Now, applying (i) at the function $u-\varepsilon$ on $\Omega_{R}$, it follows $u \leq \varepsilon$ on $\Omega$ so that, letting $\varepsilon \rightarrow 0$, (ii) is proved.

We shall prove next proposition by using the properties of the kernel $K$.

Proposition 4.2. Let $u: \mathbf{R}^{N+1} \longrightarrow[-\infty, \infty[$ be an u.s.c. function satisfying the solid sub-mean property. If $u$ is finite at $z_{0}=\left(x_{0}, t_{0}\right)$, then $u>-\infty$ in a dense subset of $\left\{(x, t) \in \mathbf{R}^{N+1} \mid t<t_{0}\right\}$. 
Proof. Let $z_{0} \in \mathrm{R}^{N+1}$ be such that $u\left(z_{0}\right)>-\infty$. By contradiction we assume that $E:=\left\{(x, t) \in \mathrm{R}^{N+1} \mid t<t_{0}, u(x, t)=-\infty\right\}$ has non-empty interior. Then, there exists $r>0$ and an open set $\Omega \subseteq E$ such that $\Omega \subseteq \Omega_{r}\left(z_{0}\right)$. Since $K\left(z_{0}, \cdot\right)>0$ in a dense open subset of $\left\{(x, t) \in \mathrm{R}^{N+1} \mid t<t_{0}\right\}$ and by the continuity of $K$, we deduce that there exists an open set $\Omega^{\prime} \subseteq \Omega$ with $K\left(z_{0}, \cdot\right)>0$ on $\Omega^{\prime}$. But this is in contradiction with

$$
-\infty<u\left(z_{0}\right) \leq M_{r}(u)\left(z_{0}\right)=\frac{1}{r^{Q-2}} \int_{\Omega_{r}\left(z_{0}\right)} K\left(\zeta^{-1} \circ z_{0}\right) u(\zeta) \mathrm{d} \zeta,
$$

and the assertion follows.

\section{Some characterizations of $\mathscr{L}$-subharmonic functions}

The aim of this section is to give some characterizations of $\mathscr{L}$-subharmonic functions in terms of the averaging operators $\mathcal{M}_{r}$ and $M_{r}$.

For any Radon measure $\mu$ in $\mathbf{R}^{N+1}$, we define the $\mathscr{L}$-potential $\Gamma_{\mu}$ of $\mu$ by

$$
\Gamma_{\mu}(z):=-\int_{\mathrm{R}^{N+1}} \Gamma\left(\zeta^{-1} \circ z\right) \mathrm{d} \mu(\zeta), \quad z \in \mathrm{R}^{N+1} .
$$

If $\Gamma_{\mu}>-\infty$ in a dense subset of $\mathrm{R}^{N+1}$, using [9, Theorem 2.7-(vi)] we easily get

$$
\mathscr{L} \Gamma_{\mu}=\mu, \quad \text { in the weak sense of distributions. }
$$

An application for Fubini's theorem shows that $\Gamma_{\mu}$ is $\mathscr{L}$-subharmonic in $\mathrm{R}^{N+1}$. Moreover, we have $\Gamma_{\mu} \in \mathscr{H}^{\mathscr{L}}\left(\mathrm{R}^{N+1} \backslash \operatorname{supp} \mu\right)$. Then, Remark 2.2 and the hypoellipticity of $\mathscr{L}$ yield the following theorem.

Theorem 5.1. Let $u \in \mathscr{S}^{\mathscr{L}}(\Omega)$ and let $\mu=\mathscr{L} u$ be its $\mathscr{L}$-Riesz measure. For every bounded open set $V \subseteq \bar{V} \subseteq \Omega$ there exists $h \in \mathscr{H}^{\mathscr{L}}(V)$ such that, for almost every $z \in V$,

$$
u(z)=-\int_{\bar{V}} \Gamma\left(\zeta^{-1} \circ z\right) \mathrm{d} \mu(\zeta)+h(z)
$$

In order to extend formula (3.4) to the class of $\mathscr{L}$-subharmonic functions in $\mathrm{R}^{N+1}$, first we give a weak result holding almost everywhere. For this purpose, we proceed as in [11, Theorem 1.6], by using the inequality (5.1) of [9], Theorem 5.1 and Corollary 3.5.

TheOREM 5.2 (Poisson-Jensen-type formula). Let $u \in \underline{\mathscr{S}}^{\mathscr{L}}(\Omega)$ and let $\mu=\mathscr{L} u$ be its related $\mathscr{L}$-Riesz measure. For almost every $z \in \Omega$ and $r>0$ 
with $\bar{\Omega}_{r}(z) \subseteq \Omega$, we have

$u(z)=M_{r}(u)(z)-\frac{Q-2}{r^{Q-2}} \int_{0}^{r} l^{Q-3}\left(\int_{\Omega_{l}(z)}\left(\Gamma\left(\zeta^{-1} \circ z\right)-\frac{1}{l^{Q-2}}\right) \mathrm{d} \mu(\zeta)\right) \mathrm{d} l$.

We will see later that (5.1) and (5.2) hold for all points of $\Omega$. Now we can state our main characterization of $\mathscr{L}$-subharmonic functions.

TheORem 5.3. Let $u: \Omega \longrightarrow[-\infty, \infty[$ be an u.s.c. function finite in a dense subset of $\Omega$. Then, the following statements are equivalent:

(i) $u \in \underline{\mathscr{S}}^{\mathscr{L}}(\Omega)$;

(ii) u satisfies the surface sub-mean property;

(iii) u satisfies the solid sub-mean property.

Proof. (i) $\Rightarrow$ (iii): If $u \in C(\Omega)$, we get the assertion by formula (5.2) and by $\mu=\mathscr{L} u \geq 0$. If $u$ is just $\mathscr{L}$-subharmonic, the claim follows from a standard approximation argument.

(i) $\Rightarrow$ (ii): Let $z \in \Omega$ be such that (5.2) holds, and let $\bar{\Omega}_{r}(z) \subseteq \Omega$ for a suitable $r>0$. As in the proof of [8, Theorem 1.6], we differentiate (5.2):

$$
\begin{aligned}
\frac{\mathrm{d}}{\mathrm{d} r} M_{r}(u)(z)=- & \frac{(Q-2)^{2}}{r^{Q-1}} \int_{0}^{r} l^{Q-3}\left(\int_{\Omega_{l}(z)}\left(\Gamma\left(\zeta^{-1} \circ z\right)-l^{2-Q}\right) \mathrm{d} \mu(\zeta)\right) \mathrm{d} l \\
& +\frac{Q-2}{r} \int_{\Omega_{r}(z)}\left(\Gamma\left(\zeta^{-1} \circ z\right)-r^{2-Q}\right) \mathrm{d} \mu(\zeta) .
\end{aligned}
$$

By Tonelli's theorem,

$$
\begin{aligned}
\int_{0}^{r} l^{Q-3}\left(\int_{\Omega_{l}(z)}\left(\Gamma\left(\zeta^{-1} \circ z\right)-l^{2-Q}\right) \mathrm{d} \mu(\zeta)\right) \mathrm{d} l \\
=\int_{\Omega_{r}(z)}\left(\int_{\left(\Gamma\left(\zeta^{-1} \circ z\right)\right)^{\frac{1}{2-Q}}}^{r} l^{Q-3}\left(\Gamma\left(\zeta^{-1} \circ z\right)-l^{2-Q}\right) \mathrm{d} l\right) \mathrm{d} \mu(\zeta) \\
=\int_{\Omega_{r}(z)}\left[\Gamma\left(\zeta^{-1} \circ z\right) \frac{l^{Q-2}}{Q-2}-\ln l\right]_{l=\left(\Gamma\left(\zeta^{-1} \circ z\right)\right)^{\frac{1}{2-Q}}}^{l=r} \mathrm{~d} \mu(\zeta) \\
=\frac{1}{Q-2}\left(r^{Q-2} \int_{\Omega_{r}(z)} \Gamma\left(\zeta^{-1} \circ z\right) \mathrm{d} \mu(\zeta)\right. \\
\left.\quad-\int_{\Omega_{r}(z)} \mathrm{d} \mu(\zeta)-\int_{\Omega_{r}(z)} \ln \left(r^{Q-2} \Gamma\left(\zeta^{-1} \circ z\right)\right) \mathrm{d} \mu(\zeta)\right)
\end{aligned}
$$


We insert this result in (5.3) and simplify, obtaining

$$
\frac{\mathrm{d}}{\mathrm{d} r} M_{r}(u)(z)=\frac{Q-2}{r^{Q-1}} \int_{\Omega_{r}(z)} \ln \left(r^{Q-2} \Gamma\left(\zeta^{-1} \circ z\right)\right) \mathrm{d} \mu(\zeta) \geq 0 .
$$

Hence, for a.e. $z \in \Omega$, the function $r \mapsto M_{r}(u)(z)$ is monotone non-decreasing. As $z \mapsto M_{r}(u)(z)$ is continuous, $M_{r}(u)(z)$ is non-decreasing w.r.t. $r$ for every $z \in \Omega$. On the other hand, by (3.5) we see that $r \mapsto M_{r}(u)(z)$ is locally absolutely continuous for $r>0$. Thus,

for every $z \in \Omega$, and $r>0$ with $\bar{\Omega}_{r}(z) \subseteq \Omega, \frac{\mathrm{d}}{\mathrm{d} r} M_{r}(u)(z)$ exists and is $\geq 0$.

As a consequence, using again (3.5) we get

$$
\frac{\mathrm{d}}{\mathrm{d} r} M_{r}(u)(z)=-\frac{(Q-2)^{2}}{r^{Q-1}} \int_{0}^{r} l^{Q-3} \mathcal{M}_{l}(u)(z) \mathrm{d} l+\frac{Q-2}{r} \mathcal{M}_{r}(u)(z),
$$

whence, by (5.4),

$$
M_{r}(u)(z) \leq M_{r}(u)(z), \quad \text { for every } \quad z \in \Omega .
$$

The assertion follows from the previous implication (i) $\Rightarrow$ (iii).

(ii) $\Rightarrow$ (iii): We suppose $u(z) \leq \mathcal{M}_{r}(u)(z)$ for every $\bar{\Omega}_{r}(z) \subseteq \Omega$. By a direct integration and (3.5),

$$
u(z) \leq \frac{Q-2}{r^{Q-2}} \int_{0}^{r} l^{Q-3} M_{l}(u)(z) \mathrm{d} l=M_{r}(u)(z) .
$$

(iii) $\Rightarrow$ (i): Let $V \subset \bar{V} \subset \Omega$ be an open $\mathscr{L}$-regular set and $\varphi \in C(\partial V)$ with $\varphi \geq\left. u\right|_{\partial V}$. Then the function $u-H_{\varphi}^{V}$ is u.s.c. and it satisfies the solid sub-mean property. Moreover, $\lim \sup _{V \ni z \rightarrow \zeta \in \partial V}\left(u-H_{\varphi}^{V}\right)(z) \leq 0$. Thus we can apply Theorem 4.1-(i) and we get $u \leq H_{\varphi}^{V}$ on $V$, so that $u \in \underline{\mathscr{S}}^{\mathscr{L}}(\Omega)$.

We also provide another characterization of $\mathscr{L}$-subharmonicity.

TheOREM 5.4. Let $u: \Omega \longrightarrow[-\infty, \infty[$ be an u.s.c. function finite in a dense subset of $\Omega$. The following statements are equivalent:

(i) $u \in \underline{\mathscr{S}}^{\mathscr{L}}(\Omega)$;

(ii) for every $z \in \Omega, r \mapsto M_{r}(u)(z)$ is monotone non-decreasing for $0<$ $r<\sup \left\{\rho>0 \mid \bar{\Omega}_{\rho}(z) \subseteq \Omega\right\}$ and

$$
u(z)=\lim _{r \rightarrow 0^{+}} M_{r}(u)(z) .
$$


Proof. (i) $\Rightarrow$ (ii): The first statement follows from (5.4). However, the proof is analogous to that of [11, Corollary 1.7].

(ii) $\Rightarrow(\mathrm{i})$ : Since $r \mapsto M_{r}(u)(z)$ is monotone non-decreasing and from (5.5), we get

$$
u(z) \leq M_{r}(u)(z) \quad \text { if } \quad 0<r<\sup \left\{\rho>0 \mid \bar{\Omega}_{\rho}(z) \subseteq \Omega\right\} .
$$

Then $u$ satisfies the solid sub-mean property so that, by Theorem 5.3, $u \in$ $\underline{\mathscr{S}}^{\mathscr{L}}(\Omega)$.

As a remarkable consequence of the property (ii) in the previous theorem, we have:

THEOREM 5.5. Let $u, v \in \underline{\mathscr{S}}^{\mathscr{L}}(\Omega)$. If $u \leq v$ almost everywhere in $\Omega$, then $u \leq v$ in $\Omega$. Consequently, if $u=v$ at all points where both functions are finite, then $u \equiv v$.

Proof. Let $\bar{\Omega}_{r}(z) \subseteq \Omega$. By integrating the inequality $u \leq v$ which holds a.e. in $\Omega_{r}(z)$, we get $M_{r}(u)(z) \leq M_{r}(v)(z)$, whence $u(z)=\lim _{r \rightarrow 0^{+}} M_{r}(u)(z)$ $\leq \lim _{r \rightarrow 0^{+}} M_{r}(v)(z)=v(z)$. The second assertion is a consequence of the first one, recalling that $u, v \in L_{\mathrm{loc}}^{1}(\Omega)$.

Now we can prove that the statement of Theorem 5.1 holds for every $z \in \Omega$.

TheOREM 5.6 (Riesz's Representation for $\underline{\mathscr{S}}^{\mathscr{L}}(\Omega)$ ). Let $u \in \underline{\mathscr{S}}^{\mathscr{L}}(\Omega)$ and let $\mu=\mathscr{L} u$ be the $\mathscr{L}$-Riesz measure related to $u$. For every bounded open set $V \subseteq \bar{V} \subseteq \Omega$, there exists $h \in \mathscr{H}^{\mathscr{L}}(V)$ such that

$$
u(z)=-\int_{\bar{V}} \Gamma\left(\zeta^{-1} \circ z\right) \mathrm{d} \mu(\zeta)+h(z), \quad z \in V .
$$

Moreover the couple $(\mu, h)$ is unique in $V$.

With Theorem 5.6 in hand, proceeding in the same way we have obtained Theorem 5.2 (see the proof of [11, Theorem 1.6]), we show that the PoissonJensen-type formula (5.2) is valid at every point $z$. For sake of clearness, we state the following

THEOREM 5.7 (Poisson-Jensen's formula). Let $u \in \underline{\mathscr{S}}^{\mathscr{L}}(\Omega)$ and let $\mu=$ $\mathscr{L} u$ be its related $\mathscr{L}$-Riesz measure. For every $z \in \Omega$ and $r>0$ with $\bar{\Omega}_{r}(z) \subseteq$ $\Omega$, we have

$$
u(z)=M_{r}(u)(z)-\frac{Q-2}{r^{Q-2}} \int_{0}^{r} l^{Q-3}\left(\int_{\Omega_{l}(z)}\left(\Gamma\left(\zeta^{-1} \circ z\right)-\frac{1}{l^{Q-2}}\right) \mathrm{d} \mu(\zeta)\right) \mathrm{d} l .
$$


We end this section with a proposition which says that the least $\mathscr{L}$-harmonic majorant of a $\mathscr{L}$-potential $\Gamma_{\mu}$ is the zero function.

Proposition 5.8. Let $\mu$ be a Radon measure in $\mathrm{R}^{N+1}$, and let $\Gamma_{\mu}$ be finite in a dense subset of $\mathrm{R}^{N+1}$. If $h \in \mathscr{H}^{\mathscr{L}}\left(\mathrm{R}^{N+1}\right)$ is such that $h \leq-\Gamma_{\mu}$, then $h \leq 0$ in $\mathbf{R}^{N+1}$. In particular, $\sup _{\mathrm{R}^{N+1}} \Gamma_{\mu}=0$.

Proof. We consider a sequence $\left\{K_{j}\right\}_{j}$ of compact sets with $K_{j} \subseteq K_{j+1}$ and $\bigcup_{j} K_{j}=\mathrm{R}^{N+1}$. Since $\left.\mu\right|_{K_{j}}$ is a compactly supported Radon measure, $\Gamma_{\left.\mu\right|_{K_{j}}}$ is finite a.e. in $\mathrm{R}^{N+1}$, so $\Gamma_{\left.\mu\right|_{\mathrm{R}} N+1 \backslash K_{j}}=\Gamma_{\mu}-\Gamma_{\left.\mu\right|_{K_{j}}}>-\infty$ in a dense subset of $\mathbf{R}^{N+1}$ and it is $\mathscr{L}$-subharmonic. Then,

$$
\begin{aligned}
\underline{\mathscr{S}}^{\mathscr{L}}\left(\mathrm{R}^{N+1}\right) \ni v(z):=h(z) & +\Gamma_{\left.\mu\right|_{\mathrm{R}^{N+1} \backslash K_{j}}}(z) \\
\leq & -\Gamma_{\left.\mu\right|_{K_{j}}}(z) \leq \mu\left(K_{j}\right) \cdot \sup _{\zeta \in K_{j}} \Gamma\left(\zeta^{-1} \circ z\right) \longrightarrow 0
\end{aligned}
$$

as $|z| \rightarrow \infty$, by [9, Proposition 2.8-(ii)]. Theorem 4.1-(ii) now gives $v \leq 0$ in $\mathrm{R}^{N+1}$, whence

$$
h(z) \leq \int_{\mathrm{R}^{N+1}} \chi_{\mathrm{R}^{N+1} \backslash K_{j}}(\zeta) \Gamma\left(\zeta^{-1} \circ z\right) \mathrm{d} \mu(\zeta), \quad z \in \mathrm{R}^{N+1} .
$$

For every $z$ in the dense set where $\Gamma_{\mu}$ is finite, by dominated convergence from (5.8) it follows that $h(z) \leq 0$. As $h$ is $\mathscr{L}$-harmonic and so continuous in $\mathrm{R}^{N+1}$, we have $h \leq 0$ everywhere.

Finally we show that $m:=\inf _{\mathrm{R}^{N+1}}\left(-\Gamma_{\mu}\right)=0$. Obviously $m \leq-\Gamma_{\mu}$ and the constant function $h \equiv m$ is $\mathscr{L}$-harmonic in $\mathrm{R}^{N+1}$. From the first part of the proof we get $m \leq 0$, and the claim is proved.

\section{Bounded-above $\mathscr{L}$-subharmonic functions in $\mathrm{R}^{N+1}$}

Let $u \in \mathscr{\mathscr { S }}^{\mathscr{L}}\left(\mathrm{R}^{N+1}\right)$ be such that $u\left(z_{0}\right)>-\infty$ for a suitable $z_{0} \in \mathrm{R}^{N+1}$ and $\mu=\mathscr{L} u$ be its related $\mathscr{L}$-Riesz measure. From the solid sub-mean property of $u$ in $z_{0}$ and the Poisson-Jensen formula (5.7), we get $\int_{0}^{r} l^{Q-3}\left(\int_{\Omega_{l}\left(z_{0}\right)}\left(\Gamma\left(\zeta^{-1}\right.\right.\right.$ 。 $\left.\left.\left.z_{0}\right)-l^{2-Q}\right) \mathrm{d} \mu(\zeta)\right) \mathrm{d} l<\infty$ for $r>0$, whence

$$
\int_{\Omega_{l}\left(z_{0}\right)}\left(\Gamma\left(\zeta^{-1} \circ z_{0}\right)-l^{2-Q}\right) \mathrm{d} \mu(\zeta)<\infty, \quad \text { for every } \quad l>0 .
$$

If $u$ and $\mu$ are as above and we set

$$
n\left(z_{0}, t\right):=\int_{\Omega_{t}\left(z_{0}\right)} \mathrm{d} \mu(\zeta)
$$


we obtain

(6.2)

$\int_{\left\{\zeta \mid 0<\Gamma\left(\zeta^{-1} \circ z_{0}\right)^{\left.\frac{1}{2-Q}<l\right\}}\right.}\left(\Gamma\left(\zeta^{-1} \circ z_{0}\right)-l^{2-Q}\right) \mathrm{d} \mu(\zeta)=\int_{0}^{l}\left(t^{2-Q}-l^{2-Q}\right) \mathrm{d} n\left(z_{0}, t\right)$.

As $\left\{\zeta \in \mathrm{R}^{N+1} \mid 0<\Gamma\left(\zeta^{-1} \circ z_{0}\right)^{\frac{1}{2-Q}}<l\right\}=\Omega_{l}\left(z_{0}\right)$, by (6.2) and (6.1),

$$
\int_{0}^{l}\left(t^{2-Q}-l^{2-Q}\right) \mathrm{d} n\left(z_{0}, t\right)<\infty, \quad \text { for every } \quad l>0 .
$$

Now, integrating by parts,

(6.4)

$$
\begin{aligned}
& \int_{0}^{l}\left(t^{2-Q}-l^{2-Q}\right) \mathrm{d} n\left(z_{0}, t\right) \\
& \quad=\lim _{\varepsilon \rightarrow 0^{+}}\left(-\left(\varepsilon^{2-Q}-l^{2-Q}\right) n\left(z_{0}, \varepsilon\right)-\int_{\varepsilon}^{l}(2-Q) t^{1-Q} n\left(z_{0}, t\right) \mathrm{d} t\right) \\
& \quad=(Q-2) \int_{0}^{l} \frac{n\left(z_{0}, t\right)}{t^{Q-1}} \mathrm{~d} t .
\end{aligned}
$$

Indeed, by dominated convergence we have

$$
n\left(z_{0}, 0^{+}\right):=\lim _{t \rightarrow 0^{+}} n\left(z_{0}, t\right)=\int_{\mathrm{R}^{N+1}} \lim _{t \rightarrow 0^{+}} \chi_{\Omega_{t}\left(z_{0}\right)}(\zeta) \mathrm{d} \mu(\zeta)=0,
$$

so that

$$
\begin{aligned}
\left(\varepsilon^{2-Q}-l^{2-Q}\right) n\left(z_{0}, \varepsilon\right) & =\left(\varepsilon^{2-Q}-l^{2-Q}\right)\left(n\left(z_{0}, \varepsilon\right)-n\left(z_{0}, 0^{+}\right)\right) \\
& =\left(\varepsilon^{2-Q}-l^{2-Q}\right) \lim _{t \rightarrow 0^{+}} \int_{t}^{\varepsilon} \mathrm{d} n\left(z_{0}, t\right) \\
& =\int_{0}^{\varepsilon}\left(\varepsilon^{2-Q}-l^{2-Q}\right) \mathrm{d} n\left(z_{0}, t\right) \\
& \leq \int_{0}^{\varepsilon}\left(t^{2-Q}-l^{2-Q}\right) \mathrm{d} n\left(z_{0}, t\right) \longrightarrow 0 \text { as } \varepsilon \rightarrow 0^{+},
\end{aligned}
$$

where in the last limit we have used (6.3). Then, using (6.2) and (6.4) in the last term of (5.7), we obtain

$$
\begin{array}{r}
\frac{Q-2}{r^{Q-2}} \int_{0}^{r} l^{Q-3}\left(\int_{\Omega_{l}\left(z_{0}\right)}\left(\Gamma\left(\zeta^{-1} \circ z_{0}\right)-\frac{1}{l^{Q-2}}\right) \mathrm{d} \mu(\zeta)\right) \mathrm{d} l \\
=\frac{(Q-2)^{2}}{r^{Q-2}} \int_{0}^{r} l^{Q-3}\left(\int_{0}^{l} \frac{n\left(z_{0}, t\right)}{t^{Q-1}} \mathrm{~d} t\right) \mathrm{d} l
\end{array}
$$


Now, replacing (6.5) in Poisson-Jensen's formula (5.7) we immediately get the following representation formula for $\mathscr{L}$-subharmonic functions in $\mathrm{R}^{N+1}$.

THEOREM 6.1. Let $u \in \underline{\mathscr{S}}^{\mathscr{L}}\left(\mathrm{R}^{N+1}\right)$ be such that $u\left(z_{0}\right)>-\infty$ for a suitable $z_{0} \in \mathrm{R}^{N+1}$ and $\mu=\mathscr{L} u$ be its related $\mathscr{L}$-Riesz measure. Then, for every $R>0$, we have

$$
u\left(z_{0}\right)=M_{R}(u)\left(z_{0}\right)-(Q-2)^{2} \int_{0}^{1} \tau^{Q-3}\left(\int_{0}^{R \tau} \frac{n\left(z_{0}, t\right)}{t^{Q-1}} \mathrm{~d} t\right) \mathrm{d} \tau .
$$

Now we are ready to prove our main result.

THEOREM 6.2. Let $\mu$ be a Radon measure in $\mathrm{R}^{N+1}$ and let $n(z, t)$ be defined as follows

$$
n(z, t):=\int_{\Omega_{t}(z)} \mathrm{d} \mu(\zeta), \quad z \in \mathrm{R}^{N+1}
$$

Then, a necessary and sufficient condition for $\mu$ to be the $\mathscr{L}$-Riesz measure related to a bounded-above $\mathscr{L}$-subharmonic function $u$ in $\mathrm{R}^{N+1}$ is that the following condition holds

$$
\int_{1}^{\infty} \frac{n(z, t)}{t^{Q-1}} \mathrm{~d} t<\infty
$$

for every $z$ in a dense subset of $\mathrm{R}^{N+1}$. If this condition is satisfied, then there exists $h \in \mathscr{H}^{\mathscr{L}}\left(\mathrm{R}^{N+1}\right), h \leq 0$, such that

$$
u(z)=U-\int_{\mathrm{R}^{N+1}} \Gamma\left(\zeta^{-1} \circ z\right) \mathrm{d} \mu(\zeta)+h(z), \quad z \in \mathrm{R}^{N+1},
$$

where $U<\infty$ is the least upper bound of $u$.

Proof. We prove the first statement of the theorem, beginning with the necessity part. Let $u \in \mathscr{\mathscr { S }}^{\mathscr{L}}\left(\mathrm{R}^{N+1}\right)$ be such that $\sup _{\mathrm{R}^{N+1}} u=U<\infty$, and we choose $z_{0} \in \mathrm{R}^{N+1}$ satisfying $u\left(z_{0}\right)>-\infty$. If we define $u^{+}:=\max \{u, 0\}$ and $u^{-}:=\max \{-u, 0\}$, then for every $R>0$ the following inequality holds

$$
M_{R}\left(u^{+}\right)\left(z_{0}\right) \leq \max \{U, 0\} \cdot M_{R}(1)\left(z_{0}\right)=\max \{U, 0\} .
$$

From representation formula (6.6) we obtain

$$
\begin{aligned}
(Q-2)^{2} \int_{0}^{1} \tau^{Q-3} & \left(\int_{\tau}^{R \tau} \frac{n\left(z_{0}, t\right)}{t^{Q-1}} \mathrm{~d} t\right) \mathrm{d} \tau=M_{R}\left(u^{+}\right)\left(z_{0}\right)-M_{1}\left(u^{+}\right)\left(z_{0}\right) \\
& +M_{1}\left(u^{-}\right)\left(z_{0}\right)-M_{R}\left(u^{-}\right)\left(z_{0}\right) \leq \max \{U, 0\}+M_{1}\left(u^{-}\right)\left(z_{0}\right),
\end{aligned}
$$


so that, by Beppo Levi's theorem,

$$
(Q-2)^{2} \int_{0}^{1} \tau^{Q-3}\left(\int_{\tau}^{\infty} \frac{n\left(z_{0}, t\right)}{t^{Q-1}} \mathrm{~d} t\right) \mathrm{d} \tau<\infty
$$

Hence, we get $\int_{1}^{\infty} t^{1-Q} n\left(z_{0}, t\right) \mathrm{d} t<\infty$ and, as the $\mathscr{L}$-subharmonic function $u$ is finite in a dense subset of $\mathrm{R}^{N+1}$, we obtain also (6.7).

Let us now prove the sufficiency part. Let $\mu$ be a Radon measure on $\mathbf{R}^{N+1}$ satisfying (6.7) and consider the function

$$
u(z):=-\int_{\mathrm{R}^{N+1}} \Gamma\left(\zeta^{-1} \circ z\right) \mathrm{d} \mu(\zeta)=\Gamma_{\mu}(z), \quad z \in \mathrm{R}^{N+1} .
$$

It is enough to prove that $u \in \underline{\mathscr{S}}^{\mathscr{L}}\left(\mathrm{R}^{N+1}\right), \mathscr{L} u=\mu$ in $\mathrm{R}^{N+1}$ and $\sup _{\mathrm{R}^{N+1}} u=0$. If we show that $u$ is finite in a dense subset of $\mathrm{R}^{N+1}$, then the first two statements immediately follow from what we have seen at the beginning of Section 5, and Proposition 5.8 yields $\sup _{\mathrm{R}^{N+1}} u=0$.

We consider $z_{0}=\left(x_{0}, t_{0}\right)$ satisfying (6.7). For every fixed $R>0$, we split $u$ as follows

$$
\begin{aligned}
u(z)=- & \int_{\left\{\zeta \mid \Gamma\left(\zeta^{-1} \circ z_{0}\right)^{\frac{1}{2-Q}} \leq R\right\}} \Gamma\left(\zeta^{-1} \circ z\right) \mathrm{d} \mu(\zeta) \\
& -\int_{\left\{\zeta \mid R<\Gamma\left(\zeta^{-1} \circ z_{0}\right)^{\frac{1}{2-Q}}<+\infty\right\}} \Gamma\left(\zeta^{-1} \circ z\right) \mathrm{d} \mu(\zeta) \\
& -\int_{\left\{\zeta \mid \Gamma\left(\zeta^{-1} \circ z_{0}\right)=0\right\}} \Gamma\left(\zeta^{-1} \circ z\right) \mathrm{d} \mu(\zeta) \\
=: & u_{1}^{R, z_{0}}(z)+u_{2}^{R, z_{0}}(z)+u_{3}^{z_{0}}(z) .
\end{aligned}
$$

The function $u_{1}^{R, z_{0}}$ is $\mathscr{L}$-subharmonic in $\mathrm{R}^{N+1}$ and the same property holds for

$$
u_{\lambda}^{R, z_{0}}(z):=-\int_{\left\{\zeta \mid R<\Gamma\left(\zeta^{-1} \circ z_{0}\right)^{\frac{1}{2-Q}}<\lambda\right\}} \Gamma\left(\zeta^{-1} \circ z\right) \mathrm{d} \mu(\zeta), \quad \lambda>R
$$

We have $u_{\lambda}^{R, z_{0}} \downarrow u_{2}^{R, z_{0}}$ as $\lambda \uparrow \infty$, hence $u_{2}^{R, z_{0}}$ is a u.s.c. function. Since $u_{\lambda}^{R, z_{0}}$ satisfies the solid sub-mean property and using Beppo Levi's theorem, we get

$$
u_{2}^{R, z_{0}}(z) \leq M_{r}\left(u_{2}^{R, z_{0}}\right)(z), \quad \text { for every } r>0 \text { and } z \in \mathrm{R}^{N+1} .
$$


Moreover

$$
\begin{aligned}
u_{\lambda}^{R, z_{0}}\left(z_{0}\right) & =-\int_{R}^{\lambda} t^{2-Q} \mathrm{~d} n\left(z_{0}, t\right) \\
& =-\lambda^{2-Q} n\left(z_{0}, \lambda\right)+R^{2-Q} n\left(z_{0}, R\right)-(Q-2) \int_{R}^{\lambda} \frac{n\left(z_{0}, t\right)}{t^{Q-1}} \mathrm{~d} t \\
& \geq-(Q-2) \int_{R}^{\infty} \frac{n\left(z_{0}, t\right)}{t^{Q-1}} \mathrm{~d} t .
\end{aligned}
$$

Indeed, as the function $n\left(z_{0}, \cdot\right)$ is non-decreasing we have

$$
\lambda^{2-Q} n\left(z_{0}, \lambda\right) \leq(Q-2) \int_{\lambda}^{\infty} \frac{n\left(z_{0}, t\right)}{t^{Q-1}} \mathrm{~d} t .
$$

Then, by (6.7), $u_{2}^{R, z_{0}}\left(z_{0}\right)=\lim _{\lambda \rightarrow \infty} u_{\lambda}^{R, z_{0}}\left(z_{0}\right)>-\infty$, so that, by Proposition $4.2, u_{2}^{R, z_{0}}>-\infty$ in a dense subset of $\left\{(x, t) \in \mathrm{R}^{N+1} \mid t<t_{0}\right\}$. On the other hand, since $\Gamma$ is supported in a half space, we have

$$
\left.\left.u_{3}^{z_{0}}(x, t)=0 \quad \text { in } \quad \mathrm{R}^{N} \times\right]-\infty, t_{0}\right] .
$$

Thus the function $u$ is finite in a dense subset of $\left\{(x, t) \in \mathrm{R}^{N+1} \mid t<t_{0}\right\}$, so that, by hypothesis (6.7), $u>-\infty$ in a dense subset of $\mathbf{R}^{N+1}$. The first assertion of the theorem is so proved.

Now, let us consider a function $v \in \mathscr{\mathscr { S }}^{\mathscr{L}}\left(\mathrm{R}^{N+1}\right)$ such that $\sup _{\mathrm{R}^{N+1}} v=U<$ $\infty$. Let $\mu=\mathscr{L} v$ be its related $\mathscr{L}$-Riesz measure. By the first part of the theorem, also the function

$$
u(z):=U+\Gamma_{\mu}(z), \quad z \in \mathrm{R}^{N+1}
$$

is $\mathscr{L}$-subharmonic in $\mathrm{R}^{N+1}$ with least upper bound $U$ and related $\mathscr{L}$-Riesz measure $\mu$. Then in the weak sense of distributions we have $\mathscr{L}(v-u)=0$. Since $\mathscr{L}$ is hypoelliptic, there exists a function $h, \mathscr{L}$-harmonic in $\mathrm{R}^{N+1}$, such that $h=v-u$ almost everywhere in $\mathbf{R}^{N+1}$. So, we get $h \leq-\Gamma_{\mu}$ a.e. in $\mathbf{R}^{N+1}$, hence everywhere as a consequence of Theorem 5.5. Now Proposition 5.8 yields $h \leq 0$ in $\mathbf{R}^{N+1}$. This completes the proof of the theorem.

Remark 6.3. We note that the hypotheses $\mathscr{L} h=0$ in $\mathrm{R}^{N+1}$ and $h \leq 0$ in the previous Theorem 6.2 do not imply that $h$ is a constant function, unlike the case of sub-Laplacians on Carnot groups. Indeed, for example, the function

$$
u(x, t)=-\exp \left(x_{1}+\cdots+x_{N}+N t\right), \quad x \in \mathrm{R}^{N}, t \in \mathrm{R},
$$

is non positive, non constant and satisfies the classical heat equation $\sum_{j=1}^{N} \partial_{x_{j}}^{2} u-\partial_{t} u=0$ in $\mathbf{R}^{N+1}$. 
If we suppose for $|u|$ a suitable growth condition that enable us to get a Liouville-type theorem for $\mathscr{L}$ (see [10]), we obtain a global representation formula exactly analogous to (7.7) of [4].

Corollary 6.4. Let $u$ be a bounded-above $\mathscr{L}$-subharmonic function in $\mathrm{R}^{N+1}$, and $\mu=\mathscr{L} u$ be its related $\mathscr{L}$-Riesz measure. If we suppose

$$
|u(0, t)|=O\left(t^{m}\right) \quad \text { as } t \longrightarrow \infty
$$

for some $m \geq 0$, then

$$
u(z)=U-\int_{\mathrm{R}^{N+1}} \Gamma\left(\zeta^{-1} \circ z\right) \mathrm{d} \mu(\zeta), \quad z \in \mathrm{R}^{N+1},
$$

where $U<\infty$ is the least upper bound of $u$.

Proof. From (6.8) of Theorem 6.2 it follows that

$$
u(z)=U+\Gamma_{\mu}(z)+h(z), \quad z \in \mathbf{R}^{N+1},
$$

where $h \leq 0$ is $\mathscr{L}$-harmonic in $\mathbf{R}^{N+1}$. By Proposition 5.8 we have $\sup _{\mathrm{R}^{N+1}} \Gamma_{\mu}=$ 0 , so that $-h \leq U-u$ in $\mathrm{R}^{N+1}$. In particular,

$$
0 \leq-h(0, t) \leq U-u(0, t)=O\left(t^{m}\right) \quad \text { as } \quad t \longrightarrow \infty .
$$

Then, by [10, Theorem 1.1], $h=$ const. in $\mathrm{R}^{N+1}$. But, since

$$
\sup _{\mathrm{R}^{N+1}}\left(U+\Gamma_{\mu}+h\right)=\sup _{\mathrm{R}^{N+1}} u=U=\sup _{\mathrm{R}^{N+1}}\left(U+\Gamma_{\mu}\right),
$$

we have $h \equiv 0$, and the assertion follows.

ACKNOwLEDGEMENTS. The author wishes to thank Professor E. Lanconelli for useful discussions on the topics presented here.

\section{REFERENCES}

1. Bauer, H., Harmonische Räume und ihre Potentialtheorie, Lecture Notes in Math. 22 (1966)

2. Bonfiglioli, A., Lanconelli, E., Liouville-type theorems for real sub-Laplacians, Manuscripta Math. 105 (2001), 111-124.

3. Bonfiglioli, A., Lanconelli, E., Maximum Principle on unbounded domains for sub-Laplacians: a Potential Theory approach, Proc. Amer. Math. Soc. 130 (2002), 2295-2304.

4. Bonfiglioli, A., Lanconelli, E., Subharmonic functions on Carnot groups, Math. Ann. 325 (2003), 97-122.

5. Bony, J.-M., Principe du maximum, inégalité de Harnack et unicité du problème de Cauchy pour les opérateurs elliptiques dégénérés, Ann. Inst. Fourier (Grenoble) 19 (1969), 277304. 
6. Constantinescu, C., Cornea, A., Potential Theory on Harmonic Spaces, Springer-Verlag, Berlin, 1972.

7. Garofalo, N., Lanconelli, E., Asymptotic behavior of fundamental solutions and potential theory of parabolic operators with variable coefficients, Math. Ann. 283 (1989), 211-239.

8. Garofalo, N., Lanconelli, E., Wiener's criterion for parabolic equations with variable coefficients and its consequences, Trans. Amer. Math. Soc. 308 (1988), 811-836.

9. Kogoj, A. E., Lanconelli, E., An invariant Harnack inequality for a class of hypoelliptic ultraparabolic equations, Mediterr. J. Math. 1 (2004), 51-80.

10. Kogoj, A. E., Lanconelli, E., One-side Liouville Theorems for a class of hypoelliptic ultraparabolic equations, Contemp. Math. 368 (2005), 305-312.

11. Lanconelli, E., Pascucci, A., Superparabolic functions related to second order hypoelliptic operators, Potential Anal. 11 (1999), 303-323.

12. Lanconelli, E., Polidoro, S., On a class of hypoelliptic evolution operators, Rend. Sem. Mat. Univ. Pol. Torino 52 (1994), 29-63. Partial differential equations, II (Turin, 1993).

13. Negrini, P., Scornazzani, V., Superharmonic functions and regularity of boundary points for a class of elliptic-parabolic partial differential operators, Boll. Un. Mat. Ital. C (6) 3 (1984), no. $1,85-106$.

14. Watson, N. A., Nevanlinna's first fundamental theorem for supertemperatures, Math. Scand. 73 (1993), 49-64.

15. Watson, N. A., The representation of smooth functions in terms of the fundamental solution of a linear parabolic equation, Ann. Polon. Math. 75 (2000), 281-287.

16. Watson, N. A., A generalized Nevanlinna theorem for supertemperatures, Ann. Acad. Sci. Fenn. Math. 28 (2003), 35-54.

\author{
DIPARTIMENTO DI MATEMATICA \\ UNIVERSITÀ DEGLI STUDI DI BOLOGNA \\ PIAZZA DI PORTA S. DONATO, 5 \\ 40126 BOLOGNA \\ ITALY \\ E-mail: cinti@dm.unibo.it
}

